\title{
A CONFIGURAÇÃO DAS IMAGENS DOS GESTORES SOBRE O CONCEITO DE ESTRATÉGIA
}

\section{IMAGES CONFIGURATION OF THE MANAGERS ABOUT THE STRATEGY CONCEPT}

GABRIEL SPERANDIO MILAN

Doutor em Engenharia de Produção, pela Universidade Federal do Rio Grande do Sul (UFRGS).

Professor, pesquisador e coordenador de cursos de pós-graduação (lato sensu) na Universidade Caxias do Sul (UCS).

Rua Francisco Getúlio Vargas, 1.130, Caxias do Sul - RS - CEP 95070-560

E-mail:gsmilan@ucs.br

\section{DEONIR DE TONI}

Doutor em Administração pela Universidade Federal do Rio Grande do Sul (UFRGS).

Professor da Universidade de Caxias do Sul (UCS).

Alameda João Dal Sasso, 800, Bento Gonçalves - RS - CEP 95700-000

E-mail: deonirdt@terra.com.br 


\section{RESUMO}

A forma como os gestores definem e implementam estratégias também depende das imagens que eles possuem do conceito de estratégia. Assim, este trabalho tem como objetivo identificar as imagens de estratégia na percepção dos gestores. Entende-se que as imagens estão organizadas como uma rede de significados ou esquemas associados. Essas imagens também se organizam em torno de alguns elementos centrais, que são socialmente aceitos e compartilhados. A identificação das imagens mentais que os gestores têm a respeito de estratégia é relevante para aprofundar a compreensão das práticas organizacionais adotadas em um determinado ambiente competitivo. Com base nas cinco dimensões propostas por Mintzberg (I987) e no método de configuração da imagem (MCI), procurou-se identificar, em uma amostra de 46 gestores, qual a imagem coletiva de estratégia destes.

\section{PALAVRAS-CHAVE}

Estratégia; Dimensões de estratégia; Configuração de imagens; Organização de imagens; Percepção dos gestores.

\section{ABSTRACT}

The form as the managers defines and implements strategies also depends on the images that they have about the strategy concept. Thus, this work has as objective to identify the strategy images in the managers' perception. One understands that the images are organized as a net of meanings or projects associates and organized around some central elements that they are socially accepted and shared. The identification of the mental images that the managers have regarding strategy is excellent to deep the understanding of the organizational practical adopted in one determined surrounding competitive environment. From the five dimensions proposals for Mintzberg (I987) and of the Method of Image Configuration (MCI), it was looked to identify, in a sample of 46 managers, which its collective strategy image. 


\section{KEYWORDS}

Strategy; Strategy dimensions; Images configuration; Images organization; Managers' perception.

\section{INTRODUÇ ̃̃O}

As imagens mentais que se configuram a partir da percepção de cada indivíduo constituem o elemento cognitivo básico do pensamento humano, sendo responsáveis pelo direcionamento do comportamento das pessoas. Sabe-se que as impressões e as convicções, ou seja, a forma como o homem vê e interpreta o mundo, são os pressupostos básicos que influenciam o seu comportamento. A compreensão de como os principais gestores das empresas percebem a estratégia organizacional pode ajudar a entender como esses responsáveis pelo direcionamento das estratégias das empresas desdobram sua visão em ações, atitudes e comportamentos profissionais, contribuindo, assim, para melhor prever os pressupostos que conduzem a competição dentro de determinada indústria ou ambiente competitivo.

Muitas das ações estratégicas das organizações são direcionadas pelas imagens mentais que seus dirigentes fazem da conjuntura geral de seus negócios e do que seja o comportamento estratégico. No entanto, identificar formas de mensurar os modelos mentais desponta como um desafio para muitos pesquisadores. Com a preocupação de apresentar uma proposta de mensuração e configuração das imagens, este trabalho tem o objetivo de identificar a imagem coletiva que alguns gestores de empresas da região da Serra Gaúcha têm em relação à estratégia organizacional, categorizando os atributos dessa imagem com base nas cinco dimensões de estratégia propostas por Mintzberg (I987), os cinco P: plano, pretexto, padrão, posição e perspectiva, os quais serão abordados na próxima seção.

Nesse contexto, foi definida a seguinte questão central de pesquisa: Qual imagem de estratégia organizacional emerge da ótica dos profissionais ligados à gestão empresarial, na região da Serra Gaúcha? Com esse propósito foram adaptadas, principalmente, a técnica de configuração de imagem de produto (TCIP), desenvolvida e evoluída por Schuler (2004) e De Toni (2005), e a técnica de configuração de imagem de serviços (TCIS), implementada por De Toni, Milan e Schuler (2005), para constituir uma nova aplicação para o método de configuração da imagem (MCI), a de configurar imagens ligadas a idéias, ou seja, imagens ideativas. 
O termo estratégia organizacional é freqüentemente empregado no cotidiano das empresas. Há, porém, uma carência de reflexão sobre a sua definição e melhores desdobramentos, o que pode contribuir para torná-lo difuso e impreciso para grande parte dos gestores (FONSECA; MACHADO-DA-SILVA, 200I). Com o nível acirrado de concorrência existente no mercado, entretanto, as empresas devem intensificar a busca de qualificação para a adoção de estratégias mais efetivas, com o propósito de estabelecer e consolidar uma vantagem competitiva sustentável, conferindo-lhes uma capacidade competitiva mais consistente e duradoura (PORTER, I985, I996; ROWE; BARNES, I998).

Essa qualificação está diretamente relacionada à forma pela qual os gestores entendem e aplicam o seu conceito de estratégia organizacional em suas empresas. Sendo assim, torna-se útil a análise de como a estratégia organizacional é compreendida pelos gestores, e o mapa de configuração de imagem (MCI) pode apresentar-se como um instrumento adequado para cumprir com essa finalidade. O MCI compõe-se de um conjunto de técnicas para explorar as imagens mentais de um determinado público sobre algum objeto.

Cabe salientar que este trabalho está organizado em quatro seções. A primeira apresenta uma revisão da literatura, definindo estratégia e a forma como as imagens se organizam na mente dos indivíduos. A segunda descreve o método de pesquisa adotado. A terceira apresenta os resultados da pesquisa, e, por fim, seguem a conclusão e algumas considerações finais.

\section{REVISÃO DA LITERATURA}

\subsection{ORGANIZAÇÃO DAS IMAGENS NA MEMÓRIA}

Dentre as diferentes perspectivas do entendimento de como as imagens estão organizadas na memória de um indivíduo, duas teorias merecem destaque: a teoria das representações sociais e a teoria do núcleo central.

A teoria das representações sociais (TRS), inicialmente abordada por Moscovici (I978), é uma das principais teorias que explicam os processos de influências sociais e como os indivíduos adquirem e organizam seus modelos mentais (BREAKWELL, 200I). Apresenta a formação dos modelos mentais como um processo socialmente mediado. Assim, o grupo de pessoas e seu comportamento, o contexto organizacional e os canais de informação são agentes fortemente influenciadores na formação das imagens dos indivíduos.

Para Moscovici (2000), as representações sociais são um conjunto de convicções, crenças e valores amplamente compartilhados pelas pessoas que têm como finalidade tornar os eventos familiares, assegurando a possibilidade de compartilhamento da rede de significados que lhes forem atribuídos. 
A TRS sugere que os modelos mentais de qualquer objeto ou fenômeno será determinado pela inclinação do indivíduo para uma forma particular de ancoragem e objetivação. É importante ter em mente que esses dois processos envolvem uma interação social e o estabelecimento de significados compartilhados e do consenso por meio da comunicação entre as pessoas (BREAKWELL, 200I). Dessa forma, se os modelos mentais de estratégia organizacional forem gerados por meio de um processo de representação social, eles serão substancialmente compartilhados pelos membros de uma subcultura discreta, particular. Isso não significa que todos os membros de uma subcultura tenham um modelo mental idêntico de estratégia organizacional, mas seus integrantes compartilham certos elementos comuns. Esses elementos comuns estão contidos no núcleo de suas representações, enquanto os elementos particularizados estão localizados nas periferias das representações do grupo.

A teoria do núcleo central, por sua vez, sugere que o homem organiza e processa as informações de forma dinâmica e evolutiva em torno de um núcleo central e de um conjunto de elementos periféricos (ABRIC, I984, I993; SÁ, I996). Segundo o pesquisador francês Jean-Claude Abric (I984), toda e qualquer representação é organizada em torno de um núcleo central, constituído de um ou mais elementos que dão à representação o seu significado e que ocupam, na estrutura dessa representação, uma posição privilegiada.

O núcleo central é determinado, em parte, pela natureza do objeto representado e, em parte, pela relação que o sujeito, ou um grupo de pessoas, mantém com esse objeto. Dessa forma, o núcleo central se constitui como um subconjunto da representação, composto de um ou mais elementos, cuja ausência desestruturaria a representação ou lhe daria uma significação completamente diferente (SÁ, I996).

Os atributos que compõem o núcleo central são marcados pela memória coletiva, e são estáveis e resistentes à mudança. Sua função é gerar significação para as imagens. Em torno do sistema central da imagem, há o sistema periférico, constituído pelos atributos mais flexíveis, sensíveis ao contexto imediato. Sua função é permitir a adaptação à realidade, bem como a diferenciação do conteúdo e a proteção ao sistema central. Os sistemas periféricos estão mais próximos das práticas do cotidiano e sujeitos às mudanças (SÁ, I996).

Entende-se, portanto, que as imagens estão organizadas como uma rede de significados ou esquemas associados e organizadas em torno de alguns elementos centrais que são socialmente aceitos e compartilhados. Mais especificamente, o uso das imagens mentais que os gestores têm de estratégia organizacional é muito proveitoso para explicar conceitualmente a maneira como a configuração de sua realidade organizacional é estruturada, bem como para compreender melhor as estratégias e as práticas organizacionais adotadas. 


\subsection{DEFININDO ESTRATÉGIA E EXPLORANDO SUAS DIMENSÕES - OS CINCO P}

Pode-se pensar em estratégia organizacional como a busca por uma posição competitiva favorável ou ideal, caracterizada pela criação de uma posição exclusiva e valiosa, efetuando tradeoffs claros (PORTER, I985 e 200I). Nesse processo, é recomendado que os gestores reflitam se suas decisões contribuem para a geração de valor para seus clientes, se estão fundamentadas em competências e recursos singulares e se há riscos iminentes e reais de esses recursos e competências serem imitados ou substituídos pela concorrência (HOOLEY; SAUNDERS; PIERCY, 2005). Do mesmo modo, fortalecer a cadeia de valor do negócio, maximizando a performance em cada área, processo e atividade da empresa, integrando fornecedores, clientes e demais stakeholders em torno de objetivos comuns, é apontado como imprescindível para o sucesso (PORTER, I985).

Para Porter (1996), como a estratégia se baseia em atividades únicas, singulares a uma determinada empresa, a definição de estratégia está relacionada a ser diferente. Significa escolher deliberadamente um conjunto de atividades diferenciadas para possibilitar a geração e entrega de um mix único de valor aos clientes.

Eisenhardt (I999) defende que a estratégia é o processo de tomada de decisão da empresa, reforçado pela sua intuição coletiva. Já para Quinn (I980), a estratégia é o padrão ou o plano que integra objetivos e metas, políticas e uma seqüência de ações coerentes com a estrutura e recursos da empresa. Jain (2000) considera estratégia como o padrão de políticas e planos para alcançar objetivos e metas, levando em conta o negócio no qual a companhia está, ou busca estar, e o tipo de empresa que ela é, ou busca ser.

De acordo com Thompson Jr. e Strickland III (2000), a estratégia consiste em um conjunto de mudanças competitivas e abordagens comerciais focadas na melhoria do desempenho organizacional. Em outras palavras, é o desenvolvimento e a implementação de um planejamento capaz de reforçar a posição mercadológica da empresa. Consoante a isso, Matsuno e Mentzer (2000) afirmam que uma empresa escolhe sua estratégia com base no entendimento do ambiente, o que direciona a atenção da empresa para determinadas dimensões de performance, nas quais ela busca se destacar.

Considerando a complexidade intrínseca à definição de estratégia, entendese que Mintzberg (1987), ao defender a possibilidade de conceituá-la por meio de cinco dimensões, os cinco P da estratégia, trouxe uma visão menos cartesiana para a academia, sem a necessidade de haver uma única definição, "a definição correta”. De acordo com Mintzberg (I987), pela sua natureza, a estratégia 
implica uma série de significados ou dimensões. A estratégia, então, pode ser compreendida como um(a):

- $\quad$ Plano (plan): equivale a dizer que estratégia é um curso de ação, uma diretriz para lidar com uma situação. Nessa dimensão, a estratégia tem duas características essenciais: é elaborada de forma anterior à implementação das ações, desenvolvida de forma consciente e, na maioria das vezes, explicitada por meio de um planejamento formal. Assim, a estratégia pode ser vista como um plano que especifica quais escolhas e caminhos serão necessários, desenhado para assegurar que os objetivos básicos do negócio sejam alcançados.

- Pretexto (ploy): nessa dimensão, a estratégia se constitui em uma manobra específica, um truque ou estratagema, com a intenção de obter vantagem sobre um concorrente ou competidor. Por exemplo, uma corporação pode ameaçar expandir sua capacidade instalada apenas para desencorajar um competidor a construir uma nova planta. A estratégia como pretexto foca sua atenção no aspecto dinâmico e competitivo entre as organizações que disputam o mercado.

- Padrão (pattern): foca na ação e indica a(s) estratégia(s) realizada(s). Como um fluxo de ações, indica a consistência no comportamento adotado ou no padrão que caracteriza uma corrente de ações.

- Posição (position): indica o lugar no ambiente onde se concentram os recursos. É uma idéia que inclui as escolhas dos segmentos e nichos, ou a posição ocupada no mercado para evitar os competidores ou ser melhor do que eles. É uma maneira de contextualizar a empresa, inserindo-a em seu ambiente competitivo.

- Perspectiva (perspective): consiste não apenas em uma posição escolhida, desejada, sob uma perspectiva de longo prazo, mas também em uma forma de os gestores e a organização, como um todo, enxergarem o mundo e avaliarem as possibilidades da empresa perante o mercado. Algumas organizações são mais agressivas, buscando novas tecnologias e novos mercados, enquanto outras percebem o mundo como mais estável, buscando criar barreiras para se proteger da concorrência e/ou de outras ameaças potencias. A estratégia, como perspectiva, é para a organização o que a personalidade é para o indivíduo. É a forma de ver o mundo, interpretá-lo e agir sobre ele, regulando o comportamento por meio de um conjunto de normas e valores compartilhados pela organização.

Essas cinco dimensões de estratégia não são mutuamente excludentes. Ao contrário, integram-se, inter-relacionam-se e complementam-se. Tais dimensões não são somente uma base sistematizada que gera uma noção de como 
lidar com os concorrentes, mas constituem, também, um provocativo capaz de potencializar o processo de exercício estratégico, ou seja, a reflexão, a antecipação do futuro pretendido (planejamento) e a ação coletiva dentro da empresa, focada em resultados, como volume de negócios (receitas), participação de mercado, lucratividade e rentabilidade (MINTZBERG, I987, 2004; MINTZBERG; AHLSTRAND; LAMPEL, 2000; MINTZBERG et al., 2006).

\subsection{IMAGEM E SUA INFLUÊNCIA NO COMPORTAMENTO DOS ESTRATEGISTAS}

Os esquemas interpretativos, ou imagens, possuem um papel fundamental no direcionamento estratégico das organizações. Por meio das imagens, é possível moldar o pensamento e direcionar as ações estratégicas das organizações (MACHADO-DA-SILVA; FONSECA; FERNANDES, 2000).

Nesse sentido, Chapman e Ferfolja (200I) salientam que os modelos mentais não são simplesmente repositórios de aprendizado passado, mas são, também, as bases para a interpretação do que esteja acontecendo atualmente. Eles influenciam fortemente a ação das pessoas através do viés cognitivo. Esse viés tem como efeito a focalização e o enrijecimento da reflexão estratégica. Dessa forma, tem-se a tendência de tomar decisões baseadas em pressupostos já conhecidos, em princípios que evitam um contato direto com o desconhecido. Cada viés é construído pela história do indivíduo e/ou da organização. Histórias de sucesso ou de fracasso são grandes formadores de vieses para o direcionamento de futuras decisões, baseadas em pressupostos anteriores (MACHADO-DASILVA; FONSECA; FERNANDES, 2000; GENTNER, I983).

Cabe salientar que as imagens constituem os conceitos e as relações que um indivíduo utiliza para compreender as várias situações ou os ambientes em que está inserido. As imagens fornecem um ponto de referência, representando uma âncora, um começo a partir do qual se inicia a ação. As imagens atuais determinam que informações receberão mais atenção ou não, e expressam o modo como as pessoas vêem ou representam um objeto.

Assim, imagens fortemente arraigadas podem levar os gestores a não enxergar mudanças ambientais (internas e externas) relevantes e a não tomar decisões adequadas, rapidamente. Fica evidente, então, que as imagens refletem o que se conhece e como se conhece, ou seja, influenciam como o sujeito assiste a elas e como ele processa as informações que recebe sobre o contexto (RODRÍGUEZ, I996; ZALTMAN, I997; SENGE, I998; MACHADO-DA-SILVA; FONSECA; FERNANDES, 2000), impactando, em última análise, em sua atitude e ação. 


\section{MÉTODO DE PESQUISA}

\subsection{A TÉCNICA DE CONFIGURAÇÃO DE IMAGENS IDEATIVAS E OS OBJETIVOS DA PESQUISA}

Com base na questão central de pesquisa "Qual imagem de estratégia organizacional emerge da ótica dos profissionais ligados à gestão empresarial, na região da Serra Gaúcha?", é proposta a utilização de uma técnica derivada do método de configuração de imagem (MCI), como um instrumento adequado para essa finalidade.

O instrumento de pesquisa adotado no trabalho se baseia em métodos já desenvolvidos e validados, tais como Customer Window (APDG, I994) e Framework for Marketing Image Management (BARICH; KOTLER, I99I), bem como em métodos desenvolvidos por pesquisadores europeus para acessar a configuração das representações sociais (ABRIC, I984; MINAYO, I997; MOSCOVICI, I978, 2000; SÁ, I996). O desenvolvimento do MCI é resultado de muitos anos de pesquisa acadêmica, desde I998 (SAMPAIO, I998; DE TONI, I998, 2005; CAIERON JÚNIOR, I999; CHALA, 2000; SCHULER, 2000, 2004; DE TONI; SCHULER, 2002, 2004; KRAFT; NIQUE, 2002; DE TONI; MILAN; BARAZETTI, 2004; DE TONI; MILAN; SCHULER, 2005).

Neste trabalho, é explorada a possibilidade de criação de mais uma técnica específica, derivada desse método, agora direcionada à configuração de imagens ideativas, ou seja, idéias, conceitos e abstrações. Sendo assim, com uma abordagem predominantemente exploratória, o trabalho apresenta os seguintes objetivos específicos:

a) identificar os atributos salientes da imagem de estratégia organizacional, na ótica dos entrevistados (gestores de empresas da Serra Gaúcha);

b) identificar a imagem central e a periférica do conceito em estudo (estratégia organizacional);

c) classificar os atributos identificados dentre as cinco dimensões propostas por Mintzberg (1987), os cinco P; e

d) apresentar uma disposição gráfica dos resultados por meio do gráfico de configuração da imagem (GCI), permitindo a visualização da configuração da imagem de estratégia organizacional, para essa amostra. 


\subsection{AMOSTRA}

A amostra é não-probabilística, estabelecida por conveniência (MALHOTRA, 200I). Foram abordados profissionais ligados à gestão de empresas, que estão intimamente relacionados à definição e implementação de estratégias organizacionais. Tais profissionais estão ligados a 46 empresas diferentes, localizadas em uma cidade do interior do Estado do Rio Grande do Sul (RS), mais especificamente na região da Serra Gaúcha.

Foram contempladas, na pesquisa, tanto empresas do setor da indústria (29 empresas), quanto do setor de comércio e serviços (I7 empresas). Dos entrevistados, I6 são empresários (proprietários das empresas) e 30 executivos (Tabela I).

\section{TABELA I}

DESCRIÇÃO DA AMOSTRA

\begin{tabular}{cccccccccc}
\hline & \multicolumn{2}{c}{ SETOR } & \multicolumn{2}{c}{ FUNÇÃO } & \multicolumn{2}{c}{ IDADE } & \multicolumn{2}{c}{ SEXO } \\
\cline { 2 - 9 } RESULTADOS & INDÚSTRIA & $\begin{array}{c}\text { COMÉRCIO E } \\
\text { SERVIÇOS }\end{array}$ & $\begin{array}{c}\text { EMPRESÁRIO } \\
\text { (PROPRIETÁRIO) }\end{array}$ & $\begin{array}{c}\text { EXECUTIVOO } \\
\text { (P30 }\end{array}$ & $\begin{array}{c}>30 \\
\text { ANOS }\end{array}$ & ANOS & M & F \\
\hline FREQÜËNCIA & 29 & 17 & 16 & 30 & 14 & 31,1 & 34 & 11 \\
\hline$\%$ & 63 & 37 & 34,8 & 65,2 & 31,1 & 58,9 & 75,6 & 24,4 \\
\hline
\end{tabular}

Fonte: Dados provenientes da pesquisa.

\subsection{O INSTRUMENTO DE COLETA DE DADOS E A CONDUÇÃO DAS ENTREVISTAS}

A configuração do conteúdo das imagens pode ser feita por meio de entrevistas individuais, argüindo-se os entrevistados sobre suas percepções e convicções. No entanto, somente perguntas diretas, em torno do assunto pesquisado, não são suficientes, pois as pessoas geralmente não articulam plenamente seus conhecimentos, opiniões e idéias quando abordadas dessa maneira (GENTNER, 200I).

Quando se considera a natureza global ou holística da imagem, modelos de mensuração com apenas escalas fechadas, tais como hierarquias, lista de apelos e ordem de importância, necessitam ser complementados com outras abordagens, pois tais técnicas apenas captam fatos específicos e não representações, impressões e redes de significados (DICHTER, I985).

Com o intuito de substituir as perguntas diretas simples por um conjunto de estímulos capaz de revelar um pouco mais do conteúdo das imagens mentais 
dos respondentes, foi utilizado um roteiro básico de questões, com o propósito de estimular a evocação espontânea de atributos pertencentes às várias categorias de elementos que, teoricamente, compõem as imagens na perspectiva dos entrevistados, conforme mostra o Quadro I.

QUADRO I

ROTEIRO BÁSICO DE QUESTÖES

ROTEIRO BÁSICO DE QUESTÕES

TIPO DE

EVOCAÇÃO

1. Quando digo estratégia organizacional, qual a primeira coisa que lhe vem à mente?

Top of mind

2. Que outras idéias Ihe vêm à mente sobre estratégia organizacional?

Geral

3. Como você descreve a estratégia organizacional?

Cognitiva

4. Qual é o significado de estratégia organizacional na sua empresa?

Simbólica

5. Quais os sentimentos que lhe vêm à mente quando digo estratégia organizacional?

Emocional

6. Quais os benefícios que a estratégia organizacional traz para a sua empresa? Funcional

Fonte: Elaborado pelos autores, mediante revisão da literatura.

Em relação às questões utilizadas, alguns comentários são pertinentes. As questões I e 2 auxiliam na identificação dos atributos mais prontamente evocados, da memória do entrevistado, quando o objeto de pesquisa é citado, no caso, estratégia organizacional. Essas duas questões buscam verificar o tom geral da representação mental que o entrevistado tem sobre o conceito.

Segundo Poiesz (1989), quando são criadas condições de descontração para o entrevistado, assegurando-lhe que não existe resposta certa ou errada e que nada específico é esperado dele, além de uma relação leve e lúdica com a situação de pesquisa, a latência nas respostas do tipo "a primeira coisa que lhe vem à cabeça” é útil para avaliar os conceitos sem que o entrevistado possa refletir muito sobre a dimensão que está sendo julgada, garantindo, assim, um pouco mais de espontaneidade às suas respostas. Para essas duas questões iniciais, portanto, empregou-se a livre associação de idéias, que é uma das formas mais utilizadas para identificar as imagens que as pessoas formam sobre objetos ou conceitos (GUIMELLI, I994).

Quanto às questões 3 a 6 , por sua vez, buscam dar uma maior consistência à coleta de dados ao repetir o mesmo questionamento básico por meio de 
outras perspectivas (abordagens cognitiva, emocional e funcional), formando, assim, uma escala unidimensional, que se interessa em propor ao entrevistado um maior número de oportunidades de revelar os conceitos que fazem parte da configuração da sua imagem.

Com base no roteiro básico de questões (Quadro I), as entrevistas foram conduzidas de forma individual e em sessão única. A duração média das entrevistas foi de trinta minutos.

\subsection{TRATAMENTO DOS DADOS}

O tratamento dos dados foi realizado em três etapas. A primeira etapa consistiu na análise de conteúdo das respostas obtidas, observando os pressupostos apregoados por Bardin (2004). Com base na análise de conteúdo, foram listados todos os atributos mencionados pelos entrevistados. Dessa forma, foi gerado o conjunto de atributos que formam a imagem de estratégia organizacional, para a amostra em análise.

Na segunda etapa, foram levantadas a freqüência e a ordem de evocação dos atributos, conforme proposto por Abric (I984) e Vergès (I992), visando criar uma distinção entre os atributos mais próximos e os mais distantes, em relação ao termo indutor empregado (estratégia organizacional). Os atributos mais próximos são considerados pertencentes à imagem central, enquanto os mais distantes à periferia da imagem.

Para cada um dos atributos identificados, foram calculados o valor de freqüência (VF) e o valor de ordem (VO). Para calcular o VO, adotou-se o seguinte procedimento: quando o atributo foi citado em primeiro lugar, ele recebeu um valor 5; em segundo lugar, valor 4; em terceiro lugar, valor 3; em quarto lugar, valor 2; e, em quinto lugar, valor I. A partir da sexta posição de evocação, os atributos não receberam mais valor algum. Finalmente, foi calculado o valor total (VT) para cada atributo, o qual representa um somatório simples entre VF e VO.

Para a atribuição das zonas de proximidade com o termo indutor, foi utilizado como critério discriminatório o VT dos atributos. A partir do VT, aplicou-se o procedimento de "divisão em quartis". Por meio dessa divisão, foram criados quatro intervalos. O intervalo gerado pelo quarto quartil, que corresponde aos valores mais altos, acolheu os atributos considerados como pertinentes à imagem central. O terceiro quartil corresponde ao intervalo do segundo valor mais alto, configurando-se como a primeira periferia da imagem. O segundo quartil corresponde ao intervalo de terceiro valor mais alto e foi denominado segunda periferia. E, finalmente, o primeiro quartil, que corresponde ao intervalo de valores menores, que é denominado periferia da imagem. 
A terceira etapa contemplou a categorização dos atributos da imagem de estratégia organizacional com base nas dimensões propostas por Mintzberg (I987), os cinco P. Nessa etapa, também foi utilizada a análise de conteúdo por parte dos pesquisadores, e, para a ponderação dos atributos, adotou-se o critério do VT.

Os resultados identificados nessas etapas da pesquisa foram dispostos graficamente no gráfico de configuração das imagens (GCI), que apresenta três informações essenciais: os atributos que compõem as imagens, a proximidade destes com a imagem central e a dimensão ou categoria a que pertencem (um dos cinco P de Mintzberg).

\section{ANÁlise dos RESUltados}

\subsection{IDENTIFICAÇÃO DOS ATRIBUTOS E SUA PROXIMIDADE COM O TERMO INDUTOR}

Pela análise de conteúdo das respostas provenientes das entrevistas individuais, foram identificados 28 atributos. Esses atributos salientes formam a imagem de estratégia organizacional, na ótica dos entrevistados.

Com base nos atributos identificados e do cálculo de VF, VO e VT, procedeu-se à aferição dos quartis (Tabela I), possibilitando a apresentação da divisão, por áreas, de proximidade com o termo indutor, ou seja, a identificação da imagem central, primeira periferia, segunda periferia e periferia, de acordo com o Tabela 2.

\section{TABELA I}

ÁREAS DE PROXIMIDADE DOS ATRIBUTOS

EM RELAÇÃO AO TERMO INDUTOR

\begin{tabular}{ccc}
\hline QUARTIS & PROXIMIDADE COM O TERMO INDUTOR & INTERVALO DO VT \\
\hline 4 & Imagem central & VT de 216 a 40,5 \\
\hline 3 & Primeira Periferia & $<40,5$ a 23,5 \\
\hline 2 & Segunda Periferia & $<23,5$ a 14 \\
\hline 1 & Periferia & $<14$ a 2 \\
\hline
\end{tabular}

Fonte: Dados provenientes da pesquisa. 


\section{TABELA 2}

ATRIBUTOS IDENTIFICADOS E SUA PROXIMIDADE COM O TERMO INDUTOR

\begin{tabular}{|c|c|c|c|c|c|}
\hline IMAGENS & N. & ATRIBUTOS IDENTIFICADOS & VF & VO & VT \\
\hline \multirow{7}{*}{$\begin{array}{l}\text { Imagem } \\
\text { central }\end{array}$} & 1 & Sistematização: organização das atividades & 58 & 158 & 216 \\
\hline & 2 & Planejamento: pensar o futuro & 62 & 129 & 191 \\
\hline & 3 & Objetivos e metas a serem atingidos & 48 & 88 & 136 \\
\hline & 4 & Direção: curso de ação, caminho ou direcionamento & 29 & 53 & 82 \\
\hline & 5 & $\begin{array}{l}\text { Resultados: faturamento, participação de mercado, lucro e } \\
\text { rentabilidade }\end{array}$ & 35 & 26 & 61 \\
\hline & 6 & Orientação para mercado & 28 & 17 & 45 \\
\hline & 7 & Posição competitiva em relação à concorrência & 19 & 26 & 45 \\
\hline \multirow{7}{*}{$\begin{array}{l}\text { Primeira } \\
\text { periferia }\end{array}$} & 8 & Envolvimento: comprometimento de todos & 24 & 15 & 39 \\
\hline & 9 & Implementação: ação, atitude, pôr em prática & 16 & 23 & 39 \\
\hline & 10 & Desenvolvimento: crescimento e evolução do negócio & 19 & 15 & 34 \\
\hline & 11 & Melhoria interna: redução de custos, produtividade etc. & 15 & 18 & 33 \\
\hline & 12 & Direcionamento ou alocação adequada de recursos & 13 & 18 & 31 \\
\hline & 13 & Perspectiva: modelos, estruturação, normas e valores & 9 & 18 & 27 \\
\hline & 14 & Satisfação, realização & 20 & 4 & 24 \\
\hline \multirow{8}{*}{$\begin{array}{l}\text { Segunda } \\
\text { periferia }\end{array}$} & 15 & Manobra para enfrentar os concorrentes & 11 & 12 & 23 \\
\hline & 16 & Responsabilidade & 9 & 8 & 17 \\
\hline & 17 & Segurança & 12 & 5 & 17 \\
\hline & 18 & Tomada de decisão & 5 & 12 & 17 \\
\hline & 19 & Vantagem competitiva & 6 & 9 & 15 \\
\hline & 20 & Competitividade & 8 & 6 & 14 \\
\hline & 21 & Conhecimento & 9 & 5 & 14 \\
\hline & 22 & $\begin{array}{l}\text { Diagnóstico organizacional: verificação dos pontos fortes e } \\
\text { fracos, oportunidades e ameaças, busca de informações }\end{array}$ & 9 & 5 & 14 \\
\hline
\end{tabular}




\section{TABELA 2 (CONTINUAÇÃO)}

ATRIBUTOS IDENTIFICADOS E SUA

PROXIMIDADE COM O TERMO INDUTOR

\begin{tabular}{llllll}
\hline IMAGENS & \multicolumn{1}{c}{ N. ATRIBUTOS IDENTIFICADOS } & VF & VO & VT \\
\hline \multirow{2}{*}{23 Melhoria de resultados } & 7 & 5 & 12 \\
\cline { 2 - 6 } & 24 & Ferramenta de trabalho & 6 & 5 & 11 \\
\cline { 2 - 6 } Periferia & 25 & Algo necessário & 4 & 7 & 11 \\
\cline { 2 - 6 } & 26 & Controle & 5 & 1 & 6 \\
\cline { 2 - 6 } & 27 & Diferenciação & 2 & 3 & 5 \\
\cline { 2 - 6 } & 28 & Relacionamento: credibilidade e confiança & 2 & 0 & 2 \\
\hline
\end{tabular}

Fonte: Dados provenientes da pesquisa.

\subsection{CLASSIFICAÇÃO DOS ATRIBUTOS COM BASE NAS DIMENSÕES DE ESTRATÉGIA (CINCO P)}

Os atributos identificados foram classificados, mediante análise de conteúdo e julgamento dos pesquisadores, dentre as cinco dimensões de estratégia propostas por Mintzberg (I987), também denominadas cinco P (plano, pretexto, padrão, posição e perspectiva). O resultado dessa classificação é apresentado na Tabela 3. Nas duas colunas da direita, encontram-se os VT associados a cada atributo, assim como o percentual que ele representa na soma de todos os VT encontrados. Além disso, encontram-se, também, o VT associado a cada categoria de atributos, segundo a classificação de Mintzberg, bem como o percentual do VT geral dos atributos que cada categoria representa. 


\section{TABELA 3}

AGRUPAMENTO DOS ATRIBUTOS

EM SUAS RESPECTIVAS DIMENSÕES

\begin{tabular}{|c|c|c|c|}
\hline $\begin{array}{l}\text { DIMENSÕES } \\
- \text { CINCO P }\end{array}$ & ATRIBUTOS DE CADA UMA DAS DIMENSÕES & VT & $\%$ \\
\hline \multirow{5}{*}{ Plano } & Direcionamento ou alocação adequada de recursos & 31 & 2,62 \\
\hline & Direção: curso de ação, caminho ou direcionamento & 82 & 6,94 \\
\hline & Objetivos e metas a serem atingidos & 136 & 11,52 \\
\hline & Planejamento: pensar o futuro & 191 & 16,17 \\
\hline & Subtotal & 440 & 37,26 \\
\hline \multirow{5}{*}{ Pretexto } & Diferenciação & 5 & 0,42 \\
\hline & Competitividade & 14 & 1,19 \\
\hline & Manobra para enfrentar os concorrentes & 23 & 1,95 \\
\hline & Vantagem competitiva & 15 & 1,27 \\
\hline & Subtotal & 57 & 4,83 \\
\hline \multirow{7}{*}{ Padrão } & $\begin{array}{l}\text { Resultados: faturamento, participação de mercado, lucro e } \\
\text { rentabilidade }\end{array}$ & 61 & 5,17 \\
\hline & Melhoria de resultados & 12 & 1,02 \\
\hline & Melhoria interna: redução de custos, produtividade etc. & 33 & 2,79 \\
\hline & Implementação: ação, atitude, pôr em prática & 39 & 3,30 \\
\hline & Ferramenta de trabalho & 11 & 0,93 \\
\hline & Desenvolvimento: crescimento e evolução do negócio & 34 & 2,88 \\
\hline & Subtotal & 190 & 16,09 \\
\hline \multirow{6}{*}{ Posição } & Posição competitiva em relação à concorrência & 45 & 3,81 \\
\hline & Orientação para o mercado & 45 & 3,81 \\
\hline & Responsabilidade & 17 & 1,44 \\
\hline & Relacionamento: credibilidade e confiança & 2 & 0,17 \\
\hline & Tomada de decisão & 17 & 1,44 \\
\hline & Subtotal & 126 & 10,67 \\
\hline
\end{tabular}




\section{TABELA 3 (CONTINUAÇÃO)}

AGRUPAMENTO DOS ATRIBUTOS

EM SUAS RESPECTIVAS DIMENSÕES

\begin{tabular}{|c|c|c|c|}
\hline $\begin{array}{l}\text { DIMENSÕES } \\
\text { - CINCO P }\end{array}$ & ATRIBUTOS DE CADA UMA DAS DIMENSÕES & VT & $\%$ \\
\hline \multirow{11}{*}{ Perspectiva } & Perspectiva: modelos, estruturação, normas e valores & 27 & 2,29 \\
\hline & $\begin{array}{l}\text { Diagnóstico organizacional: verificação dos pontos fortes e } \\
\text { fracos, oportunidades e ameaças, busca de informação }\end{array}$ & 14 & 1,19 \\
\hline & Sistematização: organização das atividades & 216 & 18,29 \\
\hline & Satisfação, realização & 24 & 2,03 \\
\hline & Envolvimento: comprometimento de todos & 39 & 3,30 \\
\hline & Segurança & 17 & 1,44 \\
\hline & Algo necessário & 11 & 0,93 \\
\hline & Controle & 6 & 0,51 \\
\hline & Conhecimento & 14 & 1,19 \\
\hline & Subtotal & 368 & 31,16 \\
\hline & Soma dos valores totais & 1.181 & 100,00 \\
\hline
\end{tabular}

Fonte: Dados provenientes da pesquisa.

Observa-se que as dimensões Plano (37,26\%) e Perspectiva (31,16\%) concentram os maiores percentuais do VT geral dos atributos, o que quer dizer que os atributos classificados nessas categorias são os mais freqüentemente e prontamente citados pela amostra entrevistada. Segundo a teoria do núcleo central, pode-se pensar que esses atributos sejam mais representativos do entendimento comum dos profissionais entrevistados sobre estratégia organizacional. Notese que os dois atributos pertencentes ao núcleo central da imagem para esses respondentes encontram-se, cada um, numa dessas duas dimensões, sugerindo consistência nos resultados dos dois tratamentos.

As demais dimensões, Padrão (I6,09\%), Posição (Iо,67\%) e Pretexto (4,83\%) apresentam, seqüencialmente, valores menores, indicando que foram citados de forma menos freqüente e pronta. Comportam os atributos dispostos na Periferia da imagem de estratégia, com exceção do atributo Resultados, que se encontra na segunda periferia.

Em relação à composição de cada uma das dimensões (Tabela 3), é possível relacionar os principais atributos inerentes a cada uma delas. Para a dimensão 
Plano, os principais atributos são: "planejamento: pensar o futuro" (I6,I7\%) e "objetivos e metas a serem atingidos" (II,52\%). Para a dimensão Pretexto, "manobra para enfrentar os concorrentes” (I,95\%), "vantagem competitiva” (I,27\%) e "competitividade" (I,I9\%). Para a dimensão Padrão, "resultados: faturamento, participação de mercado, lucro e rentabilidade" (5,I7\%) e "implementação: ação, atitude, pôr em prática" (3,30\%). Para a dimensão Posição, "posição competitiva em relação à concorrência" e "orientação para mercado" (3,8I\% para ambos os atributos). E, por fim, a dimensão Perspectiva, "sistematização: organização das atividades" (18,29\%) e "envolvimento: comprometimento de todos" (3,30\%).

\subsection{GRÁFICO DE CONFIGURAÇÃO DA IMAGEM (GCI)}

O gráfico da configuração da imagem (GCI), apresentado na Figura I, ilustra, em uma representação gráfica, as principais informações sobre a imagem de estratégia organizacional, para esse conjunto de respondentes, que a aplicação do MCI gerou.

\section{FigurA I}

CONFIGURAÇÃO DA IMAGEM DO CONCEITO DE ESTRATÉGIA ORGANIZACIONAL

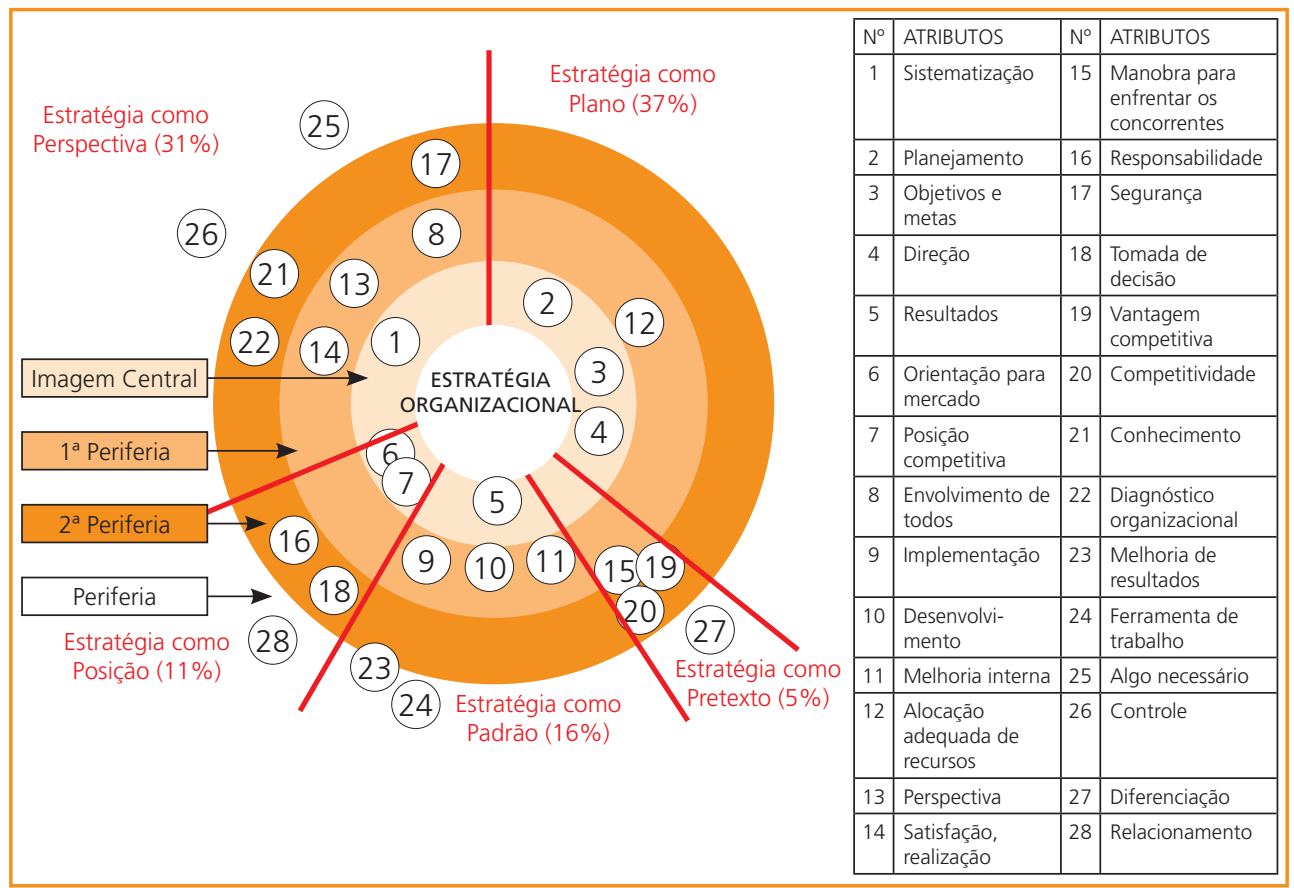

Fonte: Dados provenientes da pesquisa. 
O GCI disponibiliza três informações úteis para a visualização de imagem de estratégia organizacional: I. os atributos que compõem a imagem de estratégia organizacional; 2. as categorias de atributos, com base nos cinco P de estratégia, propostos por Mintzberg (I987); e 3. a proximidade dos atributos com o termo indutor empregado (estratégia organizacional).

O GCI de estratégia organizacional, para essa amostra, configura uma idéia cuja imagem central é constituída dos atributos (sistematização: organização das atividades e planejamento: pensar no futuro; objetivos e metas a serem atingidos; direção: curso de ação, caminho; resultados: faturamento, lucro e resultado; orientação para mercado e posição competitiva). Esses atributos são responsáveis pela sustentação da imagem compartilhada por esse grupo de gestores.

Todos os demais atributos da imagem são fugazes, encontrando-se principalmente na última periferia, o que indica que não são amplamente compartilhados, nem prontamente lembrados. Pode haver um reflexo, aqui, da forte relação nas mentes desses executivos entre estratégia e planejamento formal. Estratégia como padrão, de natureza eminentemente emergente (não deliberada) e dependente fortemente da aprendizagem organizacional (MINTZBERG; AHLSTRAND; LAMPEL, 2000), parece mais difícil de ser percebida, mesmo que existam exemplos de empresas, altamente flexíveis, que conseguiram desempenhos excelentes em ações que não foram formalmente planejadas e que as transformaram em modelos.

A adaptação de estratégias planejadas, no entanto, é muitas vezes desencorajada pela articulação de intenções e pela separação entre formulação e implementação de estratégias - várias organizações planejam não para se tornarem flexíveis, mas somente para realizar intenções específicas, e não se dão conta que, além da necessidade da direção para que as intenções se realizem, é importante responder a padrões de ação emergentes (MINTZBERG; WATERS, I985).

Em relação às dimensões de Mintzberg, a que mais se sustenta, na imagem, apesar de composta por poucos atributos, é a visão de estratégia organizacional como Plano (pensar no futuro; objetivos e metas a serem atingidos; direção: curso de ação, caminho ou direcionamento; alocação adequada de recursos). Essa dimensão comporta um conjunto de atributos mais próximos do núcleo central, perfazendo $37 \%$ do valor total encontrado no conjunto geral de atributos dessa imagem. Para um gestor que deseje prever tendências da competição nesse ambiente de negócios, seria possível supor que as organizações estarão direcionando seus planejamentos estratégicos principalmente com foco nessas questões.

A dimensão mais fracamente abordada pela amostra é a visão da estratégia como Pretexto, que comporta quatro atributos da última periferia (manobra para enfrentar concorrentes; vantagem competitiva; competitividade; diferenciação), 
perfazendo $5 \%$ do valor total encontrado no conjunto geral de atributos dessa imagem. Esse resultado indicaria aos professores e consultores da região as áreas a serem abordadas para melhor preparar os gestores para enfrentar as dificuldades de desenvolvimento de seus negócios. Tal resultado sugere, também, oportunidades de foco para quem deseja vencer nesse ambiente, uma vez que essas idéias são mais dificilmente acessadas pelos gestores da região.

Teoricamente, a diferenciação em relação aos concorrentes, por meio de um atendimento melhor das necessidades e dos desejos dos clientes, de um produto e/ou um serviço inovador ou com qualidade superior, gera um valor superior para o cliente e aumenta a possibilidade de desempenho da empresa (SONG; PARRY, I997; COOPER, 2000; HENARD; SZYMANSKI, 200I).

Da mesma forma, o conceito de relacionamento, que obteve o menor valor total do conjunto de atributos, remete para uma mudança de filosofia empresarial, para um novo modo de pensar as relações organizacionais, criando valor para o cliente, fazendo que a competitividade da empresa aumente. A habilidade da empresa em proporcionar um valor superior ao cliente, e a seus stakeholders, é considerada uma das capacidades empresariais mais direcionadas ao sucesso do negócio. Nessa perspectiva, comprador e vendedor podem ser vistos como parceiros na busca de ganhos mútuos por meio da cooperação, da confiança, da orientação de longo prazo e do comprometimento (HUNT; MORGAN, I994; GRÖNROOS, 2000; BERRY, 2002).

\section{CONCLUSÃO E CONSIDERAÇÕES FINAIS}

Apesar da natureza exploratória deste estudo, pode-se observar que os resultados são bastante interessantes no que se refere à identificação da imagem de estratégia organizacional para alguns gestores de empresas dessa região. Esses resultados sugerem que a técnica de configuração de imagens ideativas (TCID) pode ser adequada para a finalidade que pretende, convidando a testes mais profundos de validade e confiabilidade.

$\mathrm{O}$ aporte teórico de Mintzberg (1987) sobre os cinco P de estratégia, que direcionou a categorização do conceito em dimensões, contribuiu muito para formatar as imagens dos gestores entrevistados à luz desse conceito. No caso da mensuração de outras idéias, na falta de categorias assumidas a priori, por meio de teorias, pode-se utilizar a análise fatorial exploratória para verificar a formação espontânea de categorias dentro do conjunto de atributos salientes levantados na amostra.

O MCI anteriormente foi utilizado para gerar técnicas de configuração de imagens de organizações, produtos, serviços e marcas. Aqui houve um esfor- 
ço de pesquisa para gerar uma técnica de configuração de imagens de objetos puramente ideativos, tal como conceitos. Assim, a identificação das imagens de conceitos, como o de estratégia organizacional, constitui uma excelente oportunidade para melhor compreender determinados comportamentos no ambiente empresarial, possibilitando a implementação de ações para manter ou modificar a imagem existente e seus respectivos efeitos e resultados.

Dessa forma, é possível refletir sobre os modelos ou esquemas vigentes nas mentes dos executivos e de como isso pode direcionar a forma como eles enxergam seus mundos, se relacionam com seus ambientes e tomam decisões, uma vez que

são os significados atribuídos pelos indivíduos à realidade em que se acham inseridos que conformam seu contexto institucional de referência, ou seja, que definem estruturas e orientam ações organizacionais (CARVALHO; VIEIRA; GOULART, 2005, p. 865).

Nesse sentido, como afirma Morgan (2000), é preciso, para um gestor que ajuda as empresas a se adaptarem a inúmeras exigências de um mundo cada vez mais turbulento, ter consciência das imagens e dos pressupostos que determinam seu atual modo de pensar e desenvolver a capacidade de usar novas imagens e pressupostos.

\section{REFERÊENCIAS}

ABRIC, J. C. A theoretical and experimental approach to the study of social representations in a situation of interaction. In: FARR, R. M.; MOSCOVICI, S. (Ed.). Social representations. Cambridge: Cambridge University Press, I984. p. I69-183.

Central system, peripheral system: their functions and roles in the dynamics of social representations. Papers on Social Representations, v. 2, p. 75-78, I993. Disponível em: <http://www. psr.jku.at/psrindex.htm>. Acesso em: Io Sept. 2003.

APDG - Assessoria de Pesquisa e Desenvolvimento Gerencial. Customer Window: catálogo de informações. São Paulo: Rhodia S. A., I994.

BARDIN, L. Análise de conteúdo. 3. ed. Lisboa: Edições 70, 2004.

BARICH, H.; KOTLER, P. A framework for marketing image management. Sloan Management Review, v. 32, n. 2, p. 97-104, I99I.

BERRY, L. L. Relationship marketing of services - perspectivs from I983 and 2000. Journal of Relationship Marketing, v. I, n. I, p. 59-77, 2002.

BREAKWELL, G. M. Mental models and social representations of hazards: the significance of identity processes. Journal of Risk Research, v. 4, n. 4, p. 34I-35I, 200 I. 
CAIERON JÚNIOR, A. A identificação da imagem institucional do ponto de vista do funcionário. I999. Monografia (Trabalho de Conclusão do Curso de Administração)-Escola de Administração, Universidade Federal do Rio Grande do Sul, Porto Alegre, I999.

CARVALHO, C. A.; VIEIRA, M. M. F.; GOULART, S. A trajetória conservadora da teoria institucional. Revista de Administração Pública - RAP, v. 39, n. 4, p. 849-874, 2005.

CHALA, A. A universidade pública como representação social: levantamento do Núcleo Central da Representação da UFRGS entre seus estudantes de graduação. 2000. Dissertação (Mestrado)Faculdade de Biblioteconomia e Comunicação, Universidade Federal do Rio Grande do Sul, Porto Alegre, 2000.

CHAPMAN, J. A.; FERFOLJA, T. Fatal flaws: the acquisition of imperfect mental models and their use in hazardous situations. Journal of Intellectual Capital, v. 2, n. 4, p. 398-409, 200 I.

COOPER, R. G. Winning with new products: do it right. Ivey Business Journal, v. 64, n. 6, p. 54-60, 2000.

DE TONI, D. O processo de desenvolvimento de novos produtos: um estudo de caso na indústria plástica do segmento de acessórios para móveis. I998. Dissertação (Mestrado)-Escola de Administração, Universidade Federal do Rio Grande do Sul, Porto Alegre, I998.

Administração da imagem de produtos: desenvolvendo um instrumento para a configuração da imagem de produto. 2005. Tese (Doutorado)-Escola de Administração, Universidade Federal do Rio Grande do Sul, Porto Alegre, 2005.

DE TONI, D.; MILAN, G. S.; BARAZETTI, L. Imagens de serviços: um estudo exploratório sobre a configuração e organização das imagens dos serviços de fisioterapia de um plano de saúde. In: ENCONTRO ANUAL DA ANPAD, 28., 2004, Curitiba. Anais... Curitiba: Anpad, 2004.

DE TONI, D.; MILAN, G. S.; SCHULER, M. Configuração de imagens de serviços: um estudo aplicado aos serviços de fisioterapia disponibilizados por um plano de saúde. In: ENCONTRO ANUAL DA ANPAD, 29., 2005, Brasília. Anais... Brasília: Anpad, 2005.

DE TONI, D.; SCHULER, M. Gestão de imagens de produtos: uma mensuração das imagens do vinho. In: ASSEMBLÉIA DO CLADEA, 37., 2002, Porto Alegre. Anais... Porto Alegre: Cladea, 2002.

Gestão de imagem: desenvolvendo um instrumento para a configuração da imagem de produto. In: ENCONTRO ANUAL DA ANPAD, 28., 2004, Curitiba. Anais... Curitiba: Anpad, 2004.

DICHTER, E. What's in an image. Journal of Consumer Marketing, v. 2, n. I, p. 75-8I, I985.

EISENHARDT, K. M. Strategy as strategic decision making. Sloan Management Review, v. 40, n. 3, p. 65-72, I999.

FONSECA, V. S.; MACHADO-DA-SILVA, C. L. Indivíduo, organização e ambiente: bases para a conversação entre três perspectivas de estudo da estratégia em organizações. In: ENCONTRO ANUAL DA ANPAD, 25., 200I, Campinas. Anais... Campinas: Anpad, 200 I.

GENTNER D. Folowing waters or teeming crowds: mental models of electricity. In: GENTNER D.; STEVENS A. L. (Ed.). Mental models. Hillsdale: Erlbaum, I983. p. 99-I29.

Psychology of mental model. In: SMELSE, N.; BATES, P. (Ed.). International Encyclopedia of the Social and Behavioral Science. London: Elsevier, 200I. p. 9683-9687.

GRÖNROOS, C. Relationship marketing: the nordic school perspective. In: SHETH, J. N.; PARVATIYAR, A. (Eds.). Handbook of relationship marketing. Thousand Oaks: Sage Publications, 2000. Cap. 4, p. 95-II7. 
GUIMELLI, C. Transformation des représentations sociales, pratiques nouvelles et schèmes cognitifs de base. In: (Ed.). Structures et transformations des représentations sociales. Lausanne: Delachaux et Niestlé, I994. p. I7I-I98.

HENARD, D. H.; SZYMANSKI, D. M. Why some new products are more successful than others. Journal of Marketing Research, v. 38, n. 3, p. 362-375, $200 \mathrm{I}$.

HOOLEY, G. J.; SAUNDERS, J. A.; PIERCY, N. F. Estratégia de marketing e posicionamento competitivo. 3. ed. São Paulo: Prentice Hall, 2005.

HUNT, S. D.; MORGAN, R. M. Relationship marketing in the era of network competition. Marketing Management, v. 3, n. I, p. 19-28, I994.

JAIN, S. C. Marketing planning and strategy. 6. ed. Cincinnati: South-Western College, 2000.

KRAFT, S. E. R. H.; NIQUE, W. M. Desvendando o consumidor através das metáforas: uma aplicação da Zaltman Metaphor Elicitation Technique (ZMET). In: ENCONTRO ANUAL DA ANPAD, 26., 2002, Salvador. Anais... Salvador: Anpad, 2002.

MACHADO-DA-SILVA, C. L.; FONSECA, V. S.; FERNANDES, B. H. R. Cognição e institucionalização na dinâmica da mudança em organizações. In: RODRIGUES, S. B.; CUNHA, M. P. (Org.). Estudos organizacionais: novas perspectivas na administração de empresas, uma coletânea lusobrasileira. São Paulo: Iglu, 2000. p. I23-I50.

MALHOTRA, N. K. Pesquisa de marketing: uma orientação aplicada. 3. ed. Porto Alegre: Bookman, $200 I$.

MATSUNO, K.; MENTZER, J. T. The effects of strategy type on the market orientation-performance relationship. Journal of Marketing, v. 64, n. 4, p. I-I6, 2000.

MINAYO, M. O conceito de representações sociais dentro da sociologia clássica. In: JOVCHELOVITCH, S.; GUARESCHI, P. (Org.). Textos em representações sociais. Petrópolis: Vozes, I997. p. 89-III.

MINTZBERG, H. The strategy concept I: five ps for strategy. California Management Review, v. 30 , n. I, p. II-24, I987.

Ascensão e queda do planejamento estratégico. Porto Alegre: Bookman, 2004.

MINTZBERG, H.; AHLSTRAND, B.; LAMPEL, J. Safári de estratégia: um roteiro pela selva do planejamento estratégico. Porto Alegre: Bookman, 2000.

MINTZBERG, H.; WATERS, J. A. Of strategies, deliberate and emergent. Strategic Management Journal, v. 6, n. 3, p. 257-272, I985.

MINTZBERG, H. et al. O processo da estratégia: conceitos, contextos e casos selecionados. 4. ed. Porto Alegre: Bookman, 2006.

MORGAN, G. Imagens da organização. Edição executiva. São Paulo: Atlas, 2000.

MOSCOVICI. S. A representação social da psicanálise. Rio de Janeiro: Zahar, I978.

Social representation: explorations in social psychology. Cambridge: Polity Press, 2000.

POIESZ, B. C. The image concept: its place in consumer psychology. Journal of Economic Psychology,

v. Io, n. 4, p. 457-472, I989.

PORTER, M. E. Competitive advantage. New York: The Free Press, I985.

What is strategy? Harvard Business Review, v. 74, n. 6, p. 6I-78, I996.

Estratégia. In: Expo Management. São Paulo: HSM Management, 200 I.

QUINN, J. B. Strategies for change: logical incrementalismo. Homewood: Irwin, I980. 
RODRÍGUEZ, J. J. O. Imágenes mentales. Barcelona: Paidós, I996.

ROWE, W. G.; BARNES, J. G. Relationship marketing and sustained competitive advantage. Journal of Market Focused Management, v. 2, n. 3, p. 28I-297, I998.

SÁ, C. P. de. Sobre o núcleo central das representações sociais. Petrópolis: Vozes, I996.

SAMPAIO, E. F. L. Rastreamento de imagem organizacional na ótica do cliente interno. 1999. Dissertação (Mestrado)-Escola de Administração, Universidade Federal do Rio Grande do Sul, Porto Alegre, I998.

SCHULER, M. Management of the organizational image a method for the organizational image configuration 2000. Miami: PRSA Educator's Academy 2000 Research Conference, 2000.

. Management of the organizational image: a method for organizational image configuration. Corporate Reputation Review, London, v. 7, n. I, p. 37-53, Spring 2004.

SENGE, P. M. A quinta disciplina: arte e prática da organização de aprendizagem. São Paulo: Best Seller, I998.

SONG, M.; PARRY, M. E. A cross-national comparative study of new product development processes: Japan and United States. Journal of Marketing, v. 6I, n. 2, p. I-I8, I997.

THOMPSON JR., A. A.; STRICKLAND III, A. J. Planejamento estratégico. São Paulo: Pioneira, 2000.

VERGÈS, P. L'evocation de lárgent: une méthode pour la definition du noyau central dune representation. Bulletin de Psychologie, v. 45, n. 405, p. 203-209, I992.

ZALTMAN, G. Rethinking market research: putting people back. Journal of Marketing Research, v. 34, n. 4 , p. $456-472$, I997. 